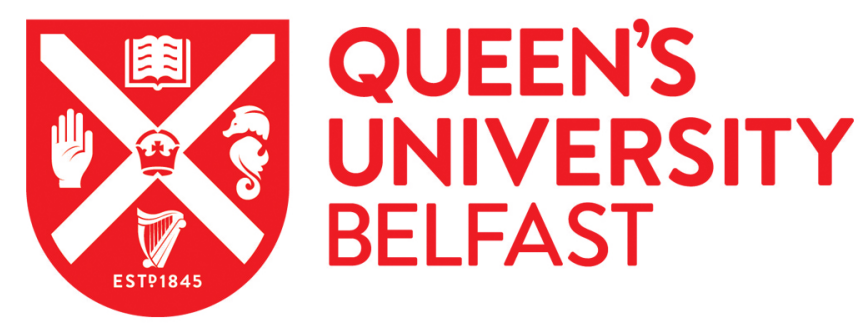

\title{
The polytropic index of solar coronal plasma in sunspot fan loops and its temperature dependence
}

Krishna Prasad, S., Raes, J. O., Van Doorsselaere, T., Magyar, N., \& Jess, D. B. (2018). The polytropic index of solar coronal plasma in sunspot fan loops and its temperature dependence. The Astrophysical Journal, 868, 1-9. [149]. https://doi.org/10.3847/1538-4357/aae9f5

\section{Published in:}

The Astrophysical Journal

Document Version:

Publisher's PDF, also known as Version of record

Queen's University Belfast - Research Portal:

Link to publication record in Queen's University Belfast Research Portal

Publisher rights

(c) 2018. The American Astronomical Society. All rights reserved.This work is made available online in accordance with the publisher's policies. Please refer to any applicable terms of use of the publisher.

\section{General rights}

Copyright for the publications made accessible via the Queen's University Belfast Research Portal is retained by the author(s) and / or other copyright owners and it is a condition of accessing these publications that users recognise and abide by the legal requirements associated with these rights.

Take down policy

The Research Portal is Queen's institutional repository that provides access to Queen's research output. Every effort has been made to ensure that content in the Research Portal does not infringe any person's rights, or applicable UK laws. If you discover content in the Research Portal that you believe breaches copyright or violates any law, please contact openaccess@qub.ac.uk. 


\title{
The Polytropic Index of Solar Coronal Plasma in Sunspot Fan Loops and Its Temperature Dependence
}

\author{
S. Krishna Prasad ${ }^{1}$ (D), J. O. Raes ${ }^{2}$, T. Van Doorsselaere ${ }^{2}$ (D), N. Magyar ${ }^{2}$, and D. B. Jess ${ }^{1,3}$ (D) \\ ${ }^{1}$ Astrophysics Research Centre, School of Mathematics and Physics, Queen’s University Belfast, Belfast, BT7 1NN, UK; krishna.prasad@qub.ac.uk \\ ${ }^{2}$ Centre for mathematical Plasma Astrophysics, KU Leuven, Celestijnenlaan 200B, 3001 Leuven, Belgium \\ ${ }^{3}$ Department of Physics and Astronomy, California State University Northridge, Northridge, CA 91330, USA \\ Received 2018 August 24; revised 2018 October 17; accepted 2018 October 18; published 2018 December 4
}

\begin{abstract}
Observations of slow magneto-acoustic waves have been demonstrated to possess a number of applications in coronal seismology. Determination of the polytropic index $(\gamma)$ is one such important application. Analyzing the amplitudes of oscillations in temperature and density corresponding to a slow magneto-acoustic wave, the polytropic index in the solar corona has been calculated and, on the basis of the obtained value, it has been inferred that thermal conduction is highly suppressed in a very hot loop, in contrast to an earlier report of high thermal conduction in a relatively colder loop. In this study, using Solar Dynamics Observatory/AIA data, we analyzed slow magneto-acoustic waves propagating along sunspot fan loops from 30 different active regions and computed polytropic indices for several loops at multiple spatial positions. The obtained $\gamma$ values vary from $1.04 \pm 0.01$ to $1.58 \pm 0.12$ and, most importantly, display a temperature dependence indicating higher $\gamma$ at hotter temperatures. This behavior brings both the previous studies to agreement, and perhaps implies a gradual suppression of thermal conduction with increase in temperature of the loop. The observed phase shifts between temperature and density oscillations, however, are substantially larger than that expected from the classical Spitzer thermal conduction, and appear to be influenced by a line-of-sight integration effect on the emission measure.
\end{abstract}

Key words: magnetohydrodynamics (MHD) - methods: observational - Sun: corona Sun: fundamental parameters

\section{Introduction}

Slow magneto-acoustic waves have been regularly observed in the solar corona since their initial discovery in polar plumes (Ofman et al. 1997, 1999; Deforest \& Gurman 1998) and coronal loops (Berghmans \& Clette 1999; De Moortel et al. 2000). Both propagating and standing versions of these waves have been found, and their properties have been extensively studied using observations and theoretical modeling (see review articles by De Moortel 2009 and Wang 2011). Standing slow waves are mainly observed in flare-related hot loop structures (Wang et al. 2002, 2005; Kumar et al. 2015; Mandal et al. 2016; Nisticò et al. 2017, see Pant et al. 2017 for an exception) and are relatively rare, whereas propagating slow waves have a photospheric source (Marsh \& Walsh 2006; Jess et al. 2012; Krishna Prasad et al. 2015) and are more common and ubiquitous in warm loops (De Moortel et al. 2002; McEwan \& de Moortel 2006; Kiddie et al. 2012; Krishna Prasad et al. 2012b, 2014). Modern high-resolution observations have significantly improved our knowledge on slow waves and have also revealed a wealth of seismological applications.

Using stereoscopic observations of slow waves from STEREO/EUVI, Marsh et al. (2009) estimated their true propagation speed and thereby deduced the temperature of the associated loop. Wang et al. (2009), employing spectroscopic observations of slow waves from EUV Imaging Spectrometer (EIS) on board Hinode (Culhane et al. 2007), obtained the inclination angle of a loop in addition to the corresponding plasma temperature. Van Doorsselaere et al. (2011) reported the measurement of polytropic index in the solar corona for the first time from the observations of slow waves using spectroscopic data from EIS. Wang et al. (2015) made a similar measurement for the plasma in a hot flare coronal loop utilizing the observations of standing slow waves. Applying the dependence of the slow wave propagation speed on the magnetic field for high plasma- $\beta$ loops, the coronal magnetic field has been estimated from both standing (Wang et al. 2007) and propagating waves (Jess et al. 2016). Utilizing the observations of accelerating slow magneto-acoustic waves in multiple channels, Krishna Prasad et al. (2017) obtained the spatial variation of temperature along a coronal loop in addition to revealing its underlying multithermal structure (King et al. 2003).

Our main focus in this study, however, is the determination of a polytropic index. There have been several studies in the past on the estimation of polytropic index using solar wind properties (e.g., Parker 1963; Roosen 1969; Kartalev et al. 2006), but our emphasis here is on the particular application of the observations of slow magneto-acoustic waves. It has been shown that thermal conduction introduces a phase lag between temperature and density perturbations of a slow magnetoacoustic wave (Owen et al. 2009). Also, from simple linearized magnetohydrodynamics (MHD) theory for slow waves, one can show that the relative amplitudes of perturbations in temperature and density are directly related through the polytropic index (Goossens 2003). Applying these, Van Doorsselaere et al. (2011) derived a polytropic index, $\gamma=1.10 \pm 0.02$, and inferred that thermal conduction is very efficient in the solar corona. The authors obtained the required temperature and density information from spectroscopic line ratios. Furthermore, through a comparison between temperature and magnetic field fluctuations found from spectropolarimetric inversions of upper-chromospheric sunspot observations, Houston et al. (2018) also uncovered a similar polytropic index, $\gamma=1.12 \pm 0.01$. Wang et al. (2015), on the other hand, 
employed a differential emission measure (DEM) analysis on broadband imaging observations of a hot flare loop exhibiting standing slow magneto-acoustic oscillations to obtain a $\gamma$ value of $1.64 \pm 0.08$, which is close to the adiabatic index $(5 / 3)$. This implies that thermal conduction is highly suppressed in this loop, in contrast to the results of Van Doorsselaere et al. (2011) and Houston et al. (2018). Extending this work, Wang et al. (2018) performed 1D MHD simulations to compare with the observations and extract further information on excitation and damping mechanisms of slow waves. In this study, we follow the approach of Wang et al. (2015) and analyze propagating slow magneto-acoustic waves in different active region fan loops using multiband imaging observations. The details of observations, obtained results, and conclusions are described in the following sections.

\section{Observations}

Fan-like coronal loops rooted in sunspots are selected from 30 different active regions observed between 2011 and 2016, for the present study. Imaging sequences of 1-hour-long durations taken in six coronal channels-namely the $94 \AA, 131 \AA, 171 \AA$ - $193 \AA$, $211 \AA$, and $335 \AA$ channels of the Atmospheric Imaging Assembly (AIA; Lemen et al. 2012) on board the Solar Dynamics Observatory (SDO; Pesnell et al. 2012) —are particularly utilized. AIA cutout data with subfields of about $180^{\prime \prime} \times 180^{\prime \prime}$ encompassing the individual fan-loop structures were obtained and processed for all the six channels using a robust pipeline developed by Rob Rutten in IDL. ${ }^{4}$ Besides applying the necessary roll angle and plate scale corrections to the downloaded level 1.0 data using aia_prep.pro (bringing them to science-grade level 1.5), this pipeline aligns images from multiple channels and corrects for any time-dependent shifts using a large subfield disk center data obtained at a lower cadence. Subpixel alignment accuracies of about 0 ." 1 are typically achieved even for the target subfields away from disk center. The spatial and temporal resolutions of the final data are about 0 ". 6 and $12 \mathrm{~s}$, respectively. Figure 1 displays the vicinity of the selected fan-loop structures from all the 30 active regions using snapshots from the AIA $171 \AA$ channel. The start times of the individual data sets along with the corresponding NOAA numbers are listed in the figure. The central coordinates, the oscillation period, and other important parameters obtained in this study are listed in Table 1.

\section{Analysis and Results}

Fan-like loop structures from each of the selected active regions were manually inspected for propagating oscillations, and a loop segment has been chosen where the oscillations show large amplitudes. These loop segments are shown as solid blue lines in Figure 1. In about five cases, we found an additional loop segment displaying oscillations with reasonably good amplitudes. These structures are marked with red solid lines in Figure 1. We constructed time-distance maps (e.g., Berghmans \& Clette 1999; De Moortel et al. 2000) for all the chosen loop segments following a method similar to that described in Krishna Prasad et al. (2012a). Although the selection of loop segments was mainly based on data from the AIA $171 \AA$ channel, similar time-distance maps were created for all six AIA coronal channels using cospatial segments. The

\footnotetext{
4 http://www.staff.science.uu.nl/ rutte101/rridl/sdolib/
}

respective intensities from all the six channels were then subjected to the regularized inversion code developed by Hannah \& Kontar (2012) to obtain the DEM at each spatial and temporal position along the loop structure.

A sample DEM profile is shown in Figure 2(c). The plus symbol in red along the loop segment shown in Figure 2(a) marks the location from where the sample profile has been extracted. As can be seen, the DEM profile is double peaked, with its first peak just under $1 \mathrm{MK}\left(\log _{10} T=6.0\right)$ and its second peak near $2 \mathrm{MK}\left(\log _{10} T=6.3\right)$ temperatures. The first peak is relatively stronger and broader, which represents the dominant emission coming from the loop, whereas the second peak appears to be likely due to the foreground/background emission. Nearly all the loop structures analyzed in this study exhibit a similar behavior. Figure 2(b) shows the temporally averaged DEM depicting its spatial variation along the loop structure. Note that the horizontal axis in this figure shows the distance along the loop, whereas the vertical axis displays the temperature in logarithmic scale. Apparently, the doublepeaked behavior is visible all along the loop and the dominant emission is coming from the low-temperature peak throughout the length, except for the bottom few arcseconds, where the foreground/background emission dominates. To properly isolate the loop emission, we employed a best-fit doubleGaussian model for the DEM profiles. The solid line in Figure 2(c) shows the obtained fit to the data, whereas the dotted lines show individual Gaussians. The temperature at which the first Gaussian peaks is then considered as a measure of the loop temperature, whereas the area under this curve provides the total emission measure. Following Sun et al. (2013) and Wang et al. (2015), we restrict the area measurement to $\pm 2 \sigma$, where $\sigma$ is the width of the Gaussian curve. The emission measure $(E M)$ and electron number density $(n)$ of the loop are related as $n=\sqrt{\frac{E M}{d}}$, where $d$ is the depth of the loop along the line of sight. Considering a symmetric cross-section for the loop, the depth is then estimated from the width of a Gaussian fitted to the crosssectional intensity profile of the loop. A suitable location is manually identified along each of the selected loop segments where the cross-sectional profile could be better fitted with a Gaussian, and the width estimated from that location is considered as the depth of the loop segment throughout its length. As it follows, our main analysis is restricted to a few arcseconds length along each loop structure, which makes this approximation reasonable. The estimated loop widths range from 3-7 AIA pixels (1-3 Mm). The density and temperature values thus obtained are used to build time-distance maps in these quantities for each of the selected loop segments.

Sample time-distance maps in intensity, temperature, and density obtained from the loop segment marked in Figure 2(a) are shown in Figure 3. The time-distance map in intensity shown here is for the data from the AIA $171 \AA$ channel. The slanted bright/dark ridges in each of these parameters highlight the compressive oscillations propagating along the loop. To enhance the visibility of these ridges, the time series at each spatial position has been filtered in the Fourier domain, suppressing oscillation power outside a narrow band around the strongest oscillation period. To achieve this, the Fourier power of the respective time series were multiplied by a normalized Gaussian centered at the oscillation period with a width of 1 minute, before applying the inverse Fourier transform that provides the filtered time series (see, e.g., Jess et al. 2017). The 


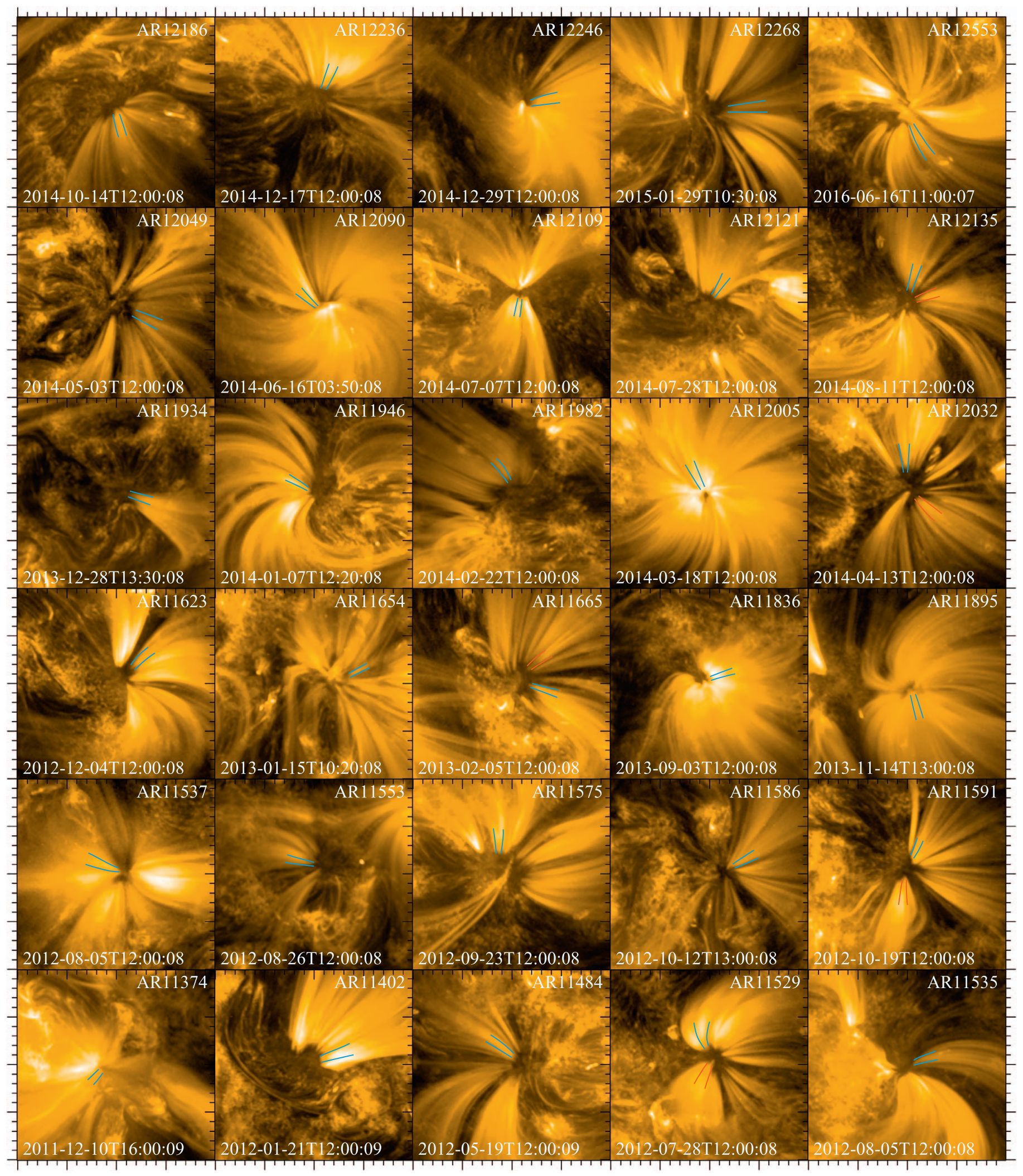

Figure 1. AIA $171 \AA$ A images displaying fan-like loop structures from 30 different active regions observed between 2011 and 2016 . The respective start times and NOAA numbers are printed on the figure. The solid lines in blue and red represent selected loop segments from individual regions. Blue and red segments correspond to "loop1," and "loop2," respectively, as listed in Table 1.

oscillation period is predetermined through a simple Fourier analysis of the AIA $171 \AA$ time series extracted from the average intensities over three adjacent pixel positions close to the bottom of the loop foot point. The average time series has been detrended to remove fluctuations of 6 minutes or longer before establishing the exact oscillation period. 
Table 1

Mean Values of Polytropic Index, Phase Shift, and Other Important Parameters for Individual Loop Structures

\begin{tabular}{|c|c|c|c|c|c|c|c|c|c|c|}
\hline $\begin{array}{l}\text { Start Time } \\
\text { (UT) }\end{array}$ & $\begin{array}{l}\text { NOAA } \\
(\#)\end{array}$ & $\begin{array}{l}\text { Location } \\
\left({ }^{\prime \prime}\right)\end{array}$ & $\begin{array}{l}\text { Loop } \\
(\#)\end{array}$ & $\begin{array}{l}P \\
(\mathrm{~s})\end{array}$ & $A_{I}$ & $\begin{array}{c}T_{0} \\
\text { (MK) }\end{array}$ & $\begin{array}{c}n_{0} \\
\left(\times 10^{9} \mathrm{~cm}^{-3}\right)\end{array}$ & $\gamma$ & $\begin{array}{c}\Delta \phi_{\text {obs }} \\
\left({ }^{\circ}\right)\end{array}$ & $\begin{array}{c}\Delta \phi_{S} \\
\left({ }^{\circ}\right)\end{array}$ \\
\hline 2011 Dec 10 16:00 & 11374 & $(-663,-281)$ & loop1 & 167 & 0.01 & $0.90 \pm 0.01$ & $2.9 \pm 0.1$ & $1.22 \pm 0.05$ & $52.3 \pm 9.5$ & 2.3 \\
\hline 2012 Jan 21 12:00 & 11402 & $(46,549)$ & loop1 & 182 & 0.05 & $0.89 \pm 0.01$ & $2.5 \pm 0.2$ & $1.04 \pm 0.01$ & $170.3 \pm 6.6$ & 0.6 \\
\hline 2012 May 19 12:00 & 11484 & $(-136,208)$ & loop1 & 198 & 0.02 & $0.88 \pm 0.01$ & $2.0 \pm 0.1$ & $1.11 \pm 0.02$ & $204.5 \pm 3.9$ & 1.5 \\
\hline \multirow[t]{2}{*}{2012 Jul 28 12:00 } & 11529 & $(-178,-269)$ & loop1 & 236 & 0.02 & $0.88 \pm 0.01$ & $1.3 \pm 0.1$ & $1.04 \pm 0.01$ & $160.7 \pm 7.2$ & 0.8 \\
\hline & & & loop2 & 153 & 0.05 & $0.90 \pm 0.01$ & $1.3 \pm 0.1$ & $1.12 \pm 0.02$ & $170.6 \pm 4.0$ & 3.5 \\
\hline 2012 Aug 5 12:00 & 11535 & $(172,221)$ & loop1 & 182 & 0.03 & $0.93 \pm 0.01$ & $0.8 \pm 0.1$ & $1.20 \pm 0.01$ & $183.3 \pm 14.8$ & 7.2 \\
\hline 2012 Aug 5 12:00 & 11537 & $(-269,115)$ & loop1 & 167 & 0.03 & $0.87 \pm 0.01$ & $1.2 \pm 0.1$ & $1.08 \pm 0.03$ & $198.1 \pm 19.8$ & 2.3 \\
\hline 2012 Aug 26 12:00 & 11553 & $(-428,-433)$ & loop1 & 182 & 0.03 & $0.97 \pm 0.01$ & $1.6 \pm 0.1$ & $1.37 \pm 0.11$ & $185.7 \pm 16.0$ & 6.3 \\
\hline 2012 Sep 23 12:00 & 11575 & $(-215,15)$ & loop1 & 236 & 0.01 & $0.91 \pm 0.02$ & $1.5 \pm 0.2$ & $1.12 \pm 0.02$ & $218.9 \pm 14.5$ & 2.1 \\
\hline 2012 Oct 12 13:00 & 11586 & $(-83,-303)$ & loop1 & 182 & 0.03 & $0.90 \pm 0.01$ & $1.4 \pm 0.1$ & $1.10 \pm 0.02$ & $195.6 \pm 19.4$ & 2.2 \\
\hline \multirow[t]{2}{*}{2012 Oct 19 12:00 } & 11591 & $(240,30)$ & loop1 & 167 & 0.09 & $0.87 \pm 0.01$ & $1.7 \pm 0.1$ & $1.05 \pm 0.01$ & $201.9 \pm 1.4$ & 1.0 \\
\hline & & & loop2 & 182 & 0.04 & $0.91 \pm 0.01$ & $1.4 \pm 0.2$ & $1.09 \pm 0.01$ & $163.1 \pm 10.7$ & 2.1 \\
\hline 2012 Dec 4 12:00 & 11623 & $(230,116)$ & loop1 & 167 & 0.04 & $0.91 \pm 0.01$ & $1.5 \pm 0.1$ & $1.11 \pm 0.01$ & $168.4 \pm 2.1$ & 2.6 \\
\hline 2013 Jan 15 10:20 & 11654 & $(310,176)$ & loop1 & 140 & 0.04 & $0.96 \pm 0.01$ & $1.8 \pm 0.1$ & $1.19 \pm 0.03$ & $186.2 \pm 6.8$ & 4.5 \\
\hline \multirow[t]{2}{*}{2013 Feb 5 12:00 } & 11665 & $(355,289)$ & loop1 & 182 & 0.06 & $0.97 \pm 0.01$ & $1.1 \pm 0.1$ & $1.16 \pm 0.03$ & $192.0 \pm 3.5$ & 5.0 \\
\hline & & & loop2 & 182 & 0.03 & $0.97 \pm 0.01$ & $0.9 \pm 0.1$ & $1.25 \pm 0.03$ & $168.8 \pm 3.8$ & 8.3 \\
\hline 2013 Sep 3 12:00 & 11836 & $(276,70)$ & loop1 & 167 & 0.03 & $0.90 \pm 0.01$ & $3.8 \pm 0.1$ & $1.11 \pm 0.01$ & $105.2 \pm 5.9$ & 1.0 \\
\hline 2013 Nov 14 13:00 & 11895 & $(-278,-316)$ & loop1 & 167 & 0.02 & $0.89 \pm 0.01$ & $2.3 \pm 0.1$ & $1.12 \pm 0.01$ & $164.1 \pm 5.9$ & 1.7 \\
\hline 2013 Dec 28 13:30 & 11934 & $(576,-215)$ & loop1 & 140 & 0.03 & $0.96 \pm 0.01$ & $1.4 \pm 0.1$ & $1.11 \pm 0.05$ & $169.8 \pm 25.9$ & 3.6 \\
\hline 2014 Jan 7 12:20 & 11946 & $(-96,220)$ & loop1 & 167 & 0.02 & $0.88 \pm 0.01$ & $2.4 \pm 0.3$ & $\ldots$ & $\ldots$ & $\cdots$ \\
\hline 2014 Feb 22 12:00 & 11982 & $(-252,-38)$ & loop1 & 182 & 0.02 & $0.93 \pm 0.01$ & $2.0 \pm 0.2$ & $1.16 \pm 0.03$ & $179.8 \pm 5.1$ & 2.5 \\
\hline 2014 Mar 18 12:00 & 12005 & $(13,317)$ & loop1 & 167 & 0.01 & $0.91 \pm 0.01$ & $4.1 \pm 0.1$ & $1.15 \pm 0.02$ & $149.6 \pm 3.1$ & 1.2 \\
\hline \multirow[t]{2}{*}{2014 Apr 13 12:00 } & 12032 & $(-68,280)$ & loop1 & 257 & 0.01 & $0.95 \pm 0.02$ & $1.2 \pm 0.2$ & $1.09 \pm 0.01$ & $197.2 \pm 6.7$ & 1.8 \\
\hline & & & loop2 & 182 & 0.03 & $0.87 \pm 0.01$ & $1.8 \pm 0.1$ & $1.11 \pm 0.04$ & $117.9 \pm 5.7$ & 1.8 \\
\hline 2014 May 3 12:00 & 12049 & $(48,-80)$ & loop1 & 198 & 0.02 & $0.93 \pm 0.01$ & $1.2 \pm 0.1$ & $1.17 \pm 0.02$ & $151.3 \pm 4.3$ & 4.1 \\
\hline 2014 Jun 16 03:50 & 12090 & $(-196,380)$ & loop1 & 182 & 0.02 & $0.94 \pm 0.02$ & $2.9 \pm 0.2$ & $\ldots$ & $\ldots$ & $\cdots$ \\
\hline 2014 Jul 07 12:00 & 12109 & $(-209,-193)$ & loop1 & 153 & 0.07 & $0.89 \pm 0.01$ & $3.2 \pm 0.2$ & $1.06 \pm 0.01$ & $161.8 \pm 15.4$ & 0.8 \\
\hline 2014 Jul 28 12:00 & 12121 & $(87,40)$ & loop1 & 167 & 0.01 & $0.88 \pm 0.01$ & $2.1 \pm 0.1$ & $1.08 \pm 0.02$ & $135.4 \pm 10.8$ & 1.3 \\
\hline \multirow[t]{2}{*}{2014 Aug 11 12:00 } & 12135 & $(33,132)$ & loop1 & 153 & 0.01 & $0.97 \pm 0.03$ & $1.3 \pm 0.1$ & $1.29 \pm 0.08$ & $187.1 \pm 11.6$ & 7.8 \\
\hline & & & loop2 & 167 & 0.02 & $1.06 \pm 0.03$ & $1.1 \pm 0.1$ & $1.55 \pm 0.04$ & $189.2 \pm 3.8$ & 14.7 \\
\hline 2014 Oct 14 12:00 & 12186 & $(166,-436)$ & loop1 & 182 & 0.02 & $0.88 \pm 0.01$ & $2.3 \pm 0.1$ & $1.07 \pm 0.01$ & $132.1 \pm 16.9$ & 1.0 \\
\hline 2014 Dec 17 12:00 & 12236 & $(-35,492)$ & loop1 & 167 & 0.04 & $0.90 \pm 0.02$ & $1.6 \pm 0.3$ & $1.09 \pm 0.01$ & $124.3 \pm 8.3$ & 2.1 \\
\hline 2014 Dec 29 12:00 & 12246 & $(347,330)$ & loop1 & 182 & 0.04 & $0.92 \pm 0.01$ & $1.3 \pm 0.1$ & $1.16 \pm 0.02$ & $171.2 \pm 8.5$ & 3.8 \\
\hline 2015 Jan 29 10:30 & 12268 & $(275,-60)$ & loop1 & 167 & 0.04 & $0.87 \pm 0.01$ & $1.4 \pm 0.1$ & $1.06 \pm 0.01$ & $171.4 \pm 9.5$ & 1.4 \\
\hline 2016 Jun 16 11:00 & 12553 & $(11,-129)$ & loop1 & 182 & 0.03 & $0.95 \pm 0.03$ & $2.1 \pm 0.1$ & $1.19 \pm 0.02$ & $180.5 \pm 21.5$ & 2.9 \\
\hline
\end{tabular}

Note. "loop1" and "loop2" are shown with blue and red solid lines, respectively, in Figure 1. $A_{I}$ denotes the relative intensity amplitudes in AIA $171 \AA$ channel, measured at the bottom of the selected loop segments.

It may be noted that the compressive oscillations decay rapidly as they propagate along the loop. Therefore, the timedistance maps presented in Figure 3 are shown only for a small section near the bottom of the loop where the amplitudes are significant. Nevertheless, as can be seen, the temperature perturbations appear to decay faster than those of the density/ intensity, a possible consequence of thermal conduction. One may also note that the amplitude of oscillations is not uniform throughout the duration. At certain times (e.g., between 20 and 35 minutes from the beginning of the time series in Figure 3), the oscillation amplitude is very much reduced in all three parameters. Therefore, using the entire time series to determine phase shifts between temperature and density perturbations will produce inaccurate results. Consequently, we restrict the phase difference calculation to a particular range in time and space where the amplitudes are large. This region is shown by the boxes in black dotted lines in Figure 3. A similar region has been manually selected for all the loop structures by visually inspecting individual time-distance maps, particularly those of temperature. The key restriction that we imposed while doing this selection is that the region should contain at least three cycles of oscillations in both temperature and density. It may be noted that, on the basis of this criterion, we could not find temperature perturbations with sufficient signal in a couple of cases, which, therefore, could not be utilized in further calculations (see Table 1).

Figure 4(a) displays relative oscillations in temperature and density within the chosen time range corresponding to the pixel position marked by a white-dashed line in Figure 3. Vertical bars, here, denote the uncertainties in respective parameters propagated from the errors on Gaussian fit to DEM curves. It may be noted that the temperature perturbations are considerably smaller than the corresponding density perturbations, as one would expect from a linearized MHD theory for slow waves. Using a cross-correlation technique, we measure the time lag $(\Delta t)$ between the two parameters and then compute the corresponding phase lag $(\Delta \phi)$ from it following the relation $\Delta \phi=(\Delta t / P) \times 360$, where $P$ is the oscillation period. The obtained phase difference in this case is about $+124^{\circ} .5$. Figure 4(b) shows a zoomed-in view of the oscillations (within the black dotted box in Figure 4(a)) with a separate scale for temperature and density to clearly highlight the observed phase difference between the quantities. The corresponding uncertainties are not shown here for clarity. 

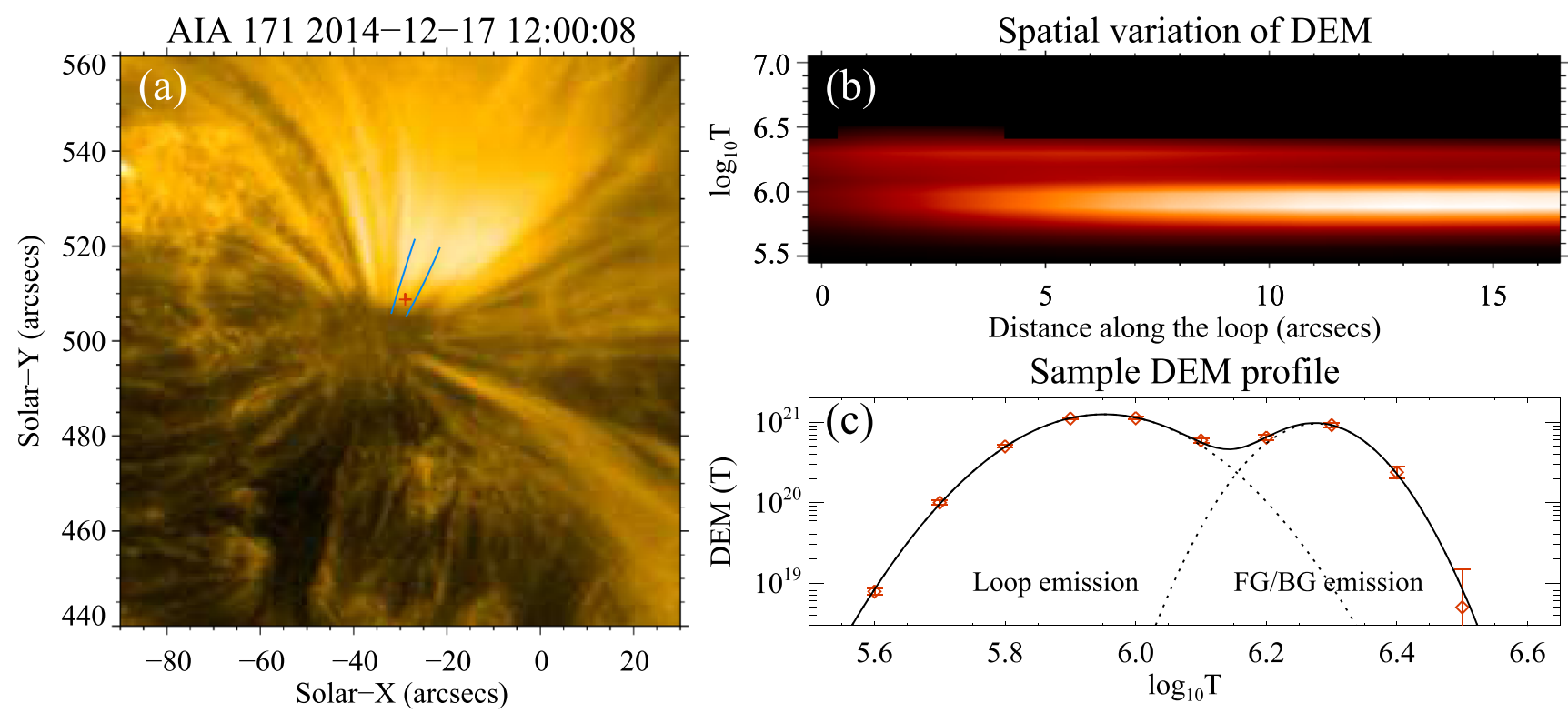

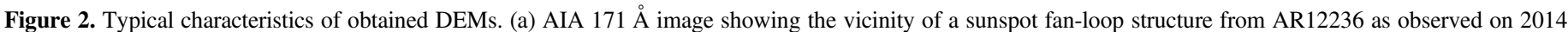

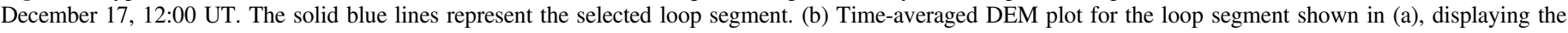

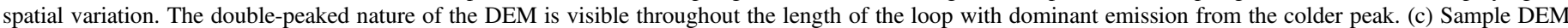

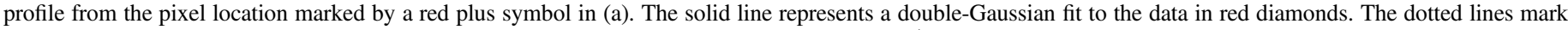
the two individual Gaussians corresponding to the emission from the loop and the foreground/background emission.

Following the linearized MHD theory for slow magnetoacoustic waves, including thermal conduction as a damping mechanism (e.g., De Moortel \& Hood 2003; Owen et al. 2009; Krishna Prasad et al. 2014), it can be shown that

$$
\begin{aligned}
& T_{\text {rel }}\left(\cos \Delta \phi+\frac{\gamma d c_{s}^{2} k^{2}}{\omega} \sin \Delta \phi-\imath \frac{\gamma d c_{s}^{2} k^{2}}{\omega} \cos \Delta \phi\right. \\
& \quad+\imath \sin \Delta \phi)=n_{\text {rel }}(\gamma-1),
\end{aligned}
$$

where $\gamma$ is the polytropic index, $\Delta \phi$ is the phase shift between density and temperature (introduced by thermal conduction), $c_{s}=\sqrt{\gamma p_{0} / \rho_{0}}$ is the sound speed, $d=\frac{(\gamma-1) k_{\|} T_{0}}{\gamma \gamma_{s}^{2} p_{0}}$ is the thermal conduction parameter, $\omega$ is the angular frequency, $k$ is the wavenumber, $T_{\text {rel }}=T^{\prime} / T_{0}$ is the relative amplitude of temperature, and $n_{\text {rel }}=n^{\prime} / n_{0}$ is the relative amplitude of density. $n_{0}, T_{0}, \rho_{0}$, and $p_{0}$ represent the equilibrium values of electron number density, temperature, mass density, and pressure, respectively. $n^{\prime}$ and $T^{\prime}$ denote the corresponding amplitudes of the perturbed values in electron number density and temperature. $k_{\|}=k_{0} T_{0}^{5 / 2}$ gives the parallel thermal conduction, where $k_{0}$ is the thermal conduction coefficient. Equating the imaginary and real parts on both sides of Equation (1) gives

$$
\begin{gathered}
\sin \Delta \phi-\frac{\gamma d c_{s}^{2} k^{2}}{\omega} \cos \Delta \phi=0, \\
T_{\text {rel }}\left(\cos \Delta \phi+\frac{\gamma d c_{s}^{2} k^{2}}{\omega} \sin \Delta \phi\right)=n_{\text {rel }}(\gamma-1) .
\end{gathered}
$$

Using the definition of $d$, and $p_{0}=2 n_{0} k_{\mathrm{B}} T_{0}$, where $k_{\mathrm{B}}$ is the Boltzmann's constant, Equation (2) can be rewritten as

$$
\tan \Delta \phi=\frac{\pi(\gamma-1) k_{\|}}{k_{\mathrm{B}} c_{s}^{2} P n_{0}}
$$

(e.g., Van Doorsselaere et al. 2011; Wang et al. 2015). Here, $P$ is the time period of the oscillation. In addition, using
Equations (2) \& (3), one may deduce

$$
T_{\text {rel }}=n_{\text {rel }}(\gamma-1) \cos \Delta \phi .
$$

Equations (4) and (5) can be used to understand the observed amplitudes of temperature and density, and the phase shifts between them. In the case of fully isothermal plasma, the polytropic index, $\gamma$, is equal to 1 ; hence, as may be inferred from Equation (5), there would not be any perturbations in temperature. On the other hand, if the conditions are perfectly adiabatic (i.e., $\gamma=5 / 3$ ) with negligible thermal conduction, Equation (4) gives $\Delta \phi \approx 0$, which implies from Equation (5) that the relative amplitude of temperature perturbations is about $66 \%(2 / 3)$ of that of density perturbations, with no phase shift between the quantities. For intermediate cases, the knowledge of the polytropic index and thermal conduction coefficient is necessary to accurately determine the values. Alternatively, one can use the observed phase shifts and amplitude values to obtain the polytropic index and the thermal conduction coefficient.

As one might note from Equations (4) and (5), a valid common solution exists only if the phase shift between temperature and density is between $0^{\circ}$ and $90^{\circ}$. However, as can be seen from Figure 4(b), the observed phase shift is about $+124^{\circ} .5$, albeit for a single spatial position along the loop structure shown in Figure 2(a). In fact, even other spatial positions and nearly all the loop structures investigated in this study show similar values well outside the expected range. This implies that there is an additional source of phase shift in observations and, consequently, that the observed values cannot be directly used to obtain the thermodynamic parameters. However, considering the phase shift to be constant over the duration of the time series, ${ }^{5}$ one can eliminate the

\footnotetext{
5 This is a valid assumption because the physical conditions on which the phase shift is dependent (see Equation (4)) do not change appreciably over the timescales involved.
} 


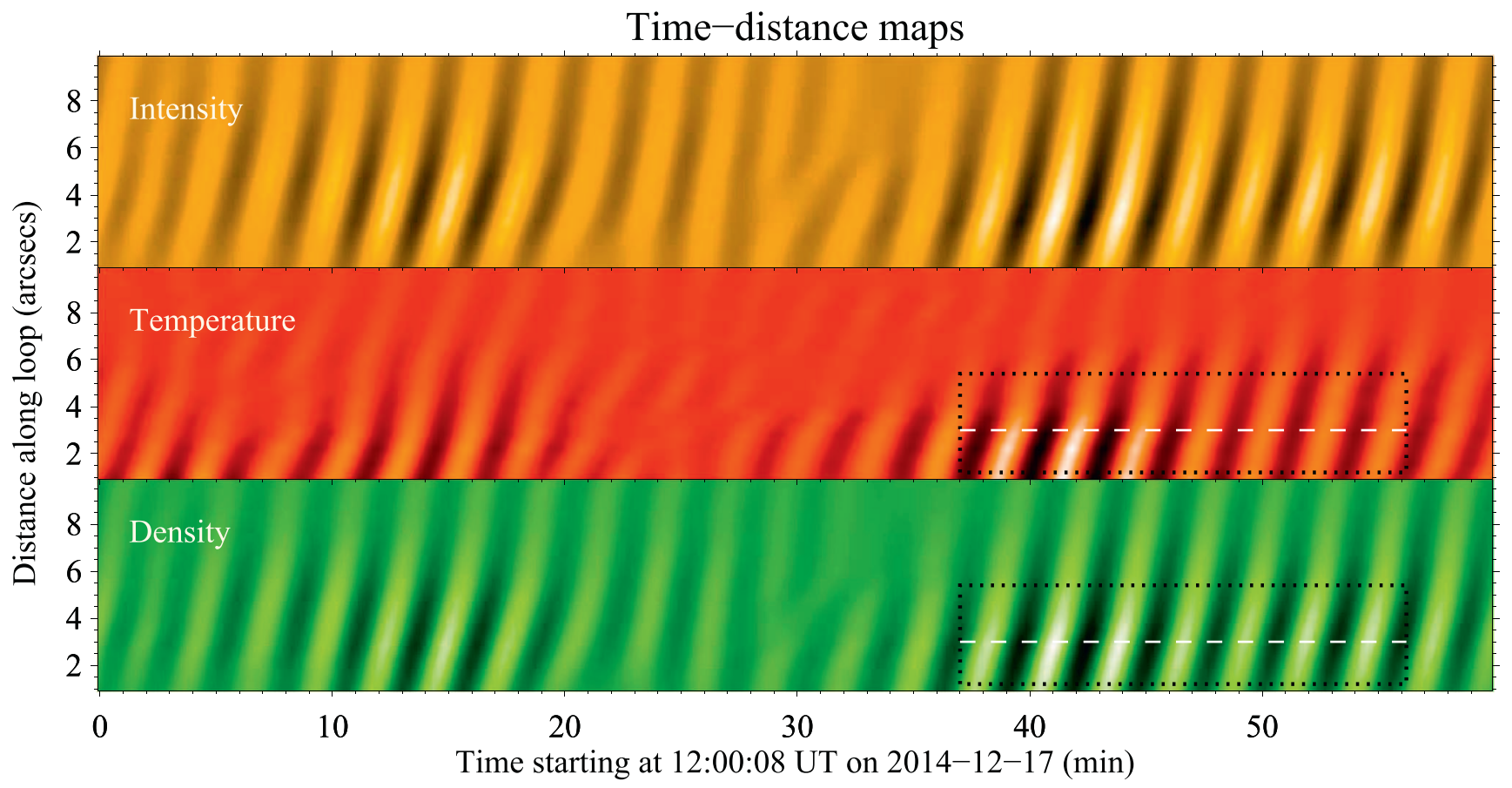

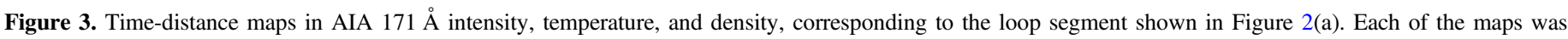

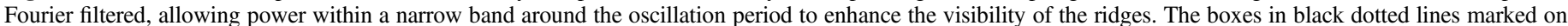

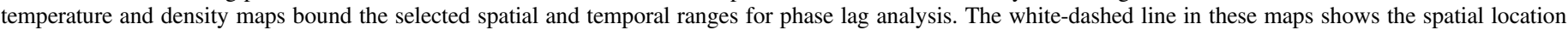
from which the temperature and density lightcurves plotted in Figure 4(a) are extracted.

dependence on it from Equation (5) by merely shifting the temperature (or density) time series to match the phase with that of density (or temperature). This step reduces Equation (5) to

$$
T_{\mathrm{rel}}^{s}=n_{\mathrm{rel}}(\gamma-1),
$$

where $\mathrm{T}_{\text {rel }}^{s}$ denotes the relative amplitudes of temperature shifted to be in phase with density. Equation (6) can then be readily used to obtain the polytropic index. In Figure 4(c), we plot $T_{\text {rel }}^{s}$ obtained by shifting the temperature perturbations shown in Figure 4(a) by $-124^{\circ} .5$ against the corresponding unshifted $n_{\text {rel }}$. The error bars here denote the respective uncertainties in temperature. A linear relationship between the parameters is evident from the data. The overplotted red solid line denotes the best linear fit obtained. Applying Equation (6), we calculate the polytropic index, $\gamma=1.10 \pm 0.01$, from the slope of the fitted line. The expected dependence for adiabatic conditions $(\gamma=5 / 3)$ is shown by a dotted line in this figure, which is largely deviated from the actual data.

It is important to note here that Equation (6) is generally applicable for slow magneto-acoustic waves whether or not there is thermal conduction. Of course, in cases of no thermal conduction, the temperature perturbations need not be shifted because they are expected to be already in phase with density. However, it does not mean that, by removing the phase shift dependence from Equation (5), we have completely eliminated the effects of thermal conduction. It is inherently assumed that the polytropic index, $\gamma$, is modified by the presence of thermal conduction, which should be reflected in the temperature and density amplitudes of a slow wave. Although this is true, in the case of coronal plasma, the polytropic index is governed not just by the thermal conduction but also by several other important processes, such as heating, radiative losses, turbulence, plasma flows, and other nonthermal processes. Therefore, a $\gamma$ value lower than 5/3, as deduced from the observed amplitudes, need not necessarily imply enhanced thermal conduction.

Keeping these limitations in mind, we computed the polytropic index at individual spatial positions across all the selected loop structures following the same procedure. The obtained values are plotted against the corresponding timeaveraged temperature (logarithmic values) in Figure 5, using diamond symbols in black. For comparison, we also show the results from Van Doorsselaere et al. (2011) and Wang et al. (2015) using a red triangle and a green square, respectively. The vertical bars in this figure represent the respective uncertainties. All our data, across 164 spatial positions identified from about 33 loop structures, are displayed in this figure. To clearly highlight the observed dependence from our data, a zoomed-in view is presented in the inset panel. Although our temperature range is limited because of our selection of only fan-like warm loop structures, it appears that the polytropic index is increasing with the temperature.

In Table 1 , we list the mean values of temperature, $T_{0}$, density, $n_{0}$, the polytropic index, $\gamma$, and the observed phase shift between the temperature and density perturbations $\left(\Delta \phi_{\text {obs }}\right)$ for all the loop structures studied. The uncertainties listed with these values are obtained from the respective standard deviations across each loop. The small uncertainties suggest that the values themselves do not vary much within a loop structure. The limited spatial ranges considered along each loop could also be partially responsible for this. It may be noted that the temperature and density values obtained here are of the same order of those found from spectroscopic observations of fan loops (Ghosh et al. 2017). The relative intensity amplitudes and the oscillation periods computed for individual loops are also listed. The amplitudes are measured from the AIA $171 \AA$ channel, near the bottom of the selected loop structures where they are usually 

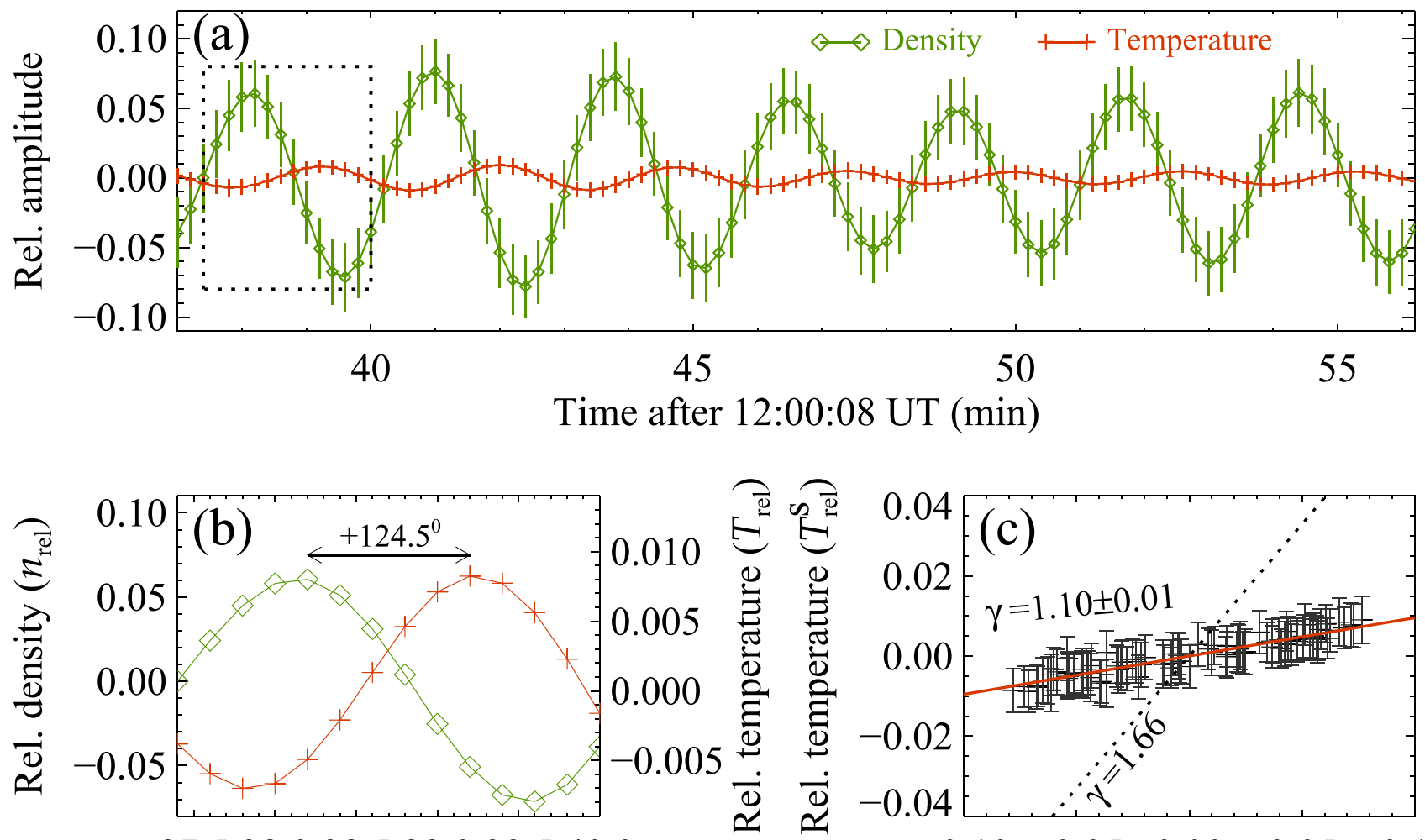

37.538 .038 .539 .039 .540 .0

Time after 12:00:08 UT (min)

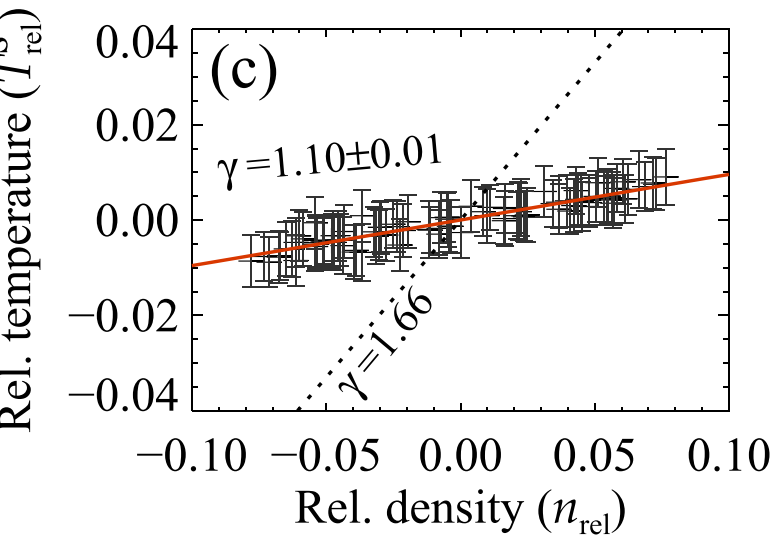

Figure 4. Determination of phase shift and polytropic index. (a) Perturbations in temperature and density from the spatial location marked by the white-dashed lines in Figure 3. The vertical bars show the respective uncertainties propagated from the errors on Gaussian fit to DEM curves. (b) A zoomed-in view of the oscillations within the black dotted box in (a), with an independent scale for density and temperature, mainly to highlight the observed phase shift between the quantities. The corresponding uncertainties are not shown here for clarity. (c) A scatter plot of relative temperature (with respective uncertainties as error bars) plotted against the relative density from (a) after shifting the temperature values to remove the existing phase shift. The red solid line represents the best linear fit to the data. The polytropic index obtained from the slope of the line is listed in the plot. The dotted line shows the expected dependence for adiabatic conditions $(\gamma=5 / 3)$.

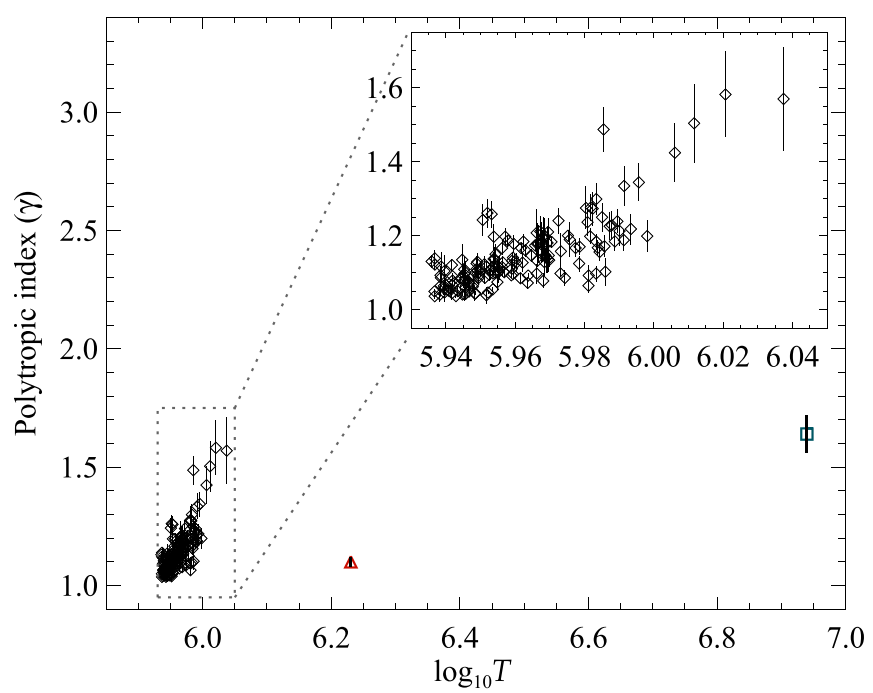

Figure 5. Dependence of polytropic index on mean temperature. Here, $\gamma$ values obtained from individual spatial positions across all the selected loop segments are plotted against the corresponding time-averaged temperature. The diamond symbols in black represent the data from this study, whereas the red triangle and the green square represent the data from Van Doorsselaere et al. (2011), and Wang et al. (2015), respectively. The vertical bars show the respective uncertainties on the values. A zoomed-in view of our data is provided in the inset panel to clearly highlight the observed dependence. the highest. The values range from 0.01 to 0.09 , suggesting the linear nature of the observed waves. A majority of the oscillation periods are near $180 \mathrm{~s}$, which is not surprising considering that the selected loop structures are rooted mainly in sunspots. In addition, in the last column, we list the expected phase shifts $\left(\Delta \phi_{S}\right)$ obtained using Equation (4), for the classical Spitzer thermal conductivity $\left(k_{0}=7.8 \times 10^{-7} ;\right.$ Spitzer 1962). The polytropic index, temperature, density, and the oscillation period used in this calculation are from the observed values. While the values $\Delta \phi_{S}$ range between 0.5 and $15^{\circ}$, the observed phase shifts, $\Delta \phi_{\text {obs }}$, vary between $52^{\circ}$ and $219^{\circ}$. Evidently, the observed phase shifts are significantly larger than the corresponding values for the classical thermal conductivity. In a couple of loops, the temperature perturbations do not possess sufficient amplitudes to estimate $\gamma, \Delta \phi_{\text {obs }}$, and $\Delta \phi_{S}$, which, therefore, are left blank.

\section{Discussion and Conclusions}

It has been demonstrated that the amplitudes of temperature and density perturbations due to a slow magneto-acoustic wave, and the phase shift introduced between them by thermal conduction, can be utilized to understand the thermodynamic properties of solar coronal plasma. Using spectroscopic data from Hinode/EIS, Van Doorsselaere et al. (2011) have 
obtained the polytropic index, $\gamma=1.10 \pm 0.02$, for a warm coronal loop and have inferred that thermal conduction is very efficient in the solar corona. More recently, Wang et al. (2015) have performed a similar analysis on a hot flare loop and obtained $\gamma=1.64 \pm 0.08$, suggesting, in contrast, a suppression in thermal conduction. Although the relevant spectroscopic data were not available, Wang et al. (2015) have extracted the required temperature and density information from broadband $S D O /$ AIA images in multiple coronal channels through DEM analysis. Following the latter approach, we studied propagating slow magneto-acoustic waves in about 30 different active regions, particularly those in fan-like loop structures. Employing a regularized inversion method (Hannah \& Kontar 2012) on the observed intensities in six AIA coronal channels, corresponding DEMs have been obtained. The DEM profiles mostly displayed a double-peaked structure, with one broader dominant peak around $1 \mathrm{MK}$ representing the loop emission and another narrow peak near 2 MK representing the foreground/background emission. We carefully isolated the loop emission using the best-fit double-Gaussian profiles and computed respective electron temperatures and densities (via emission measure) to construct time-distance maps in those parameters along selected loop segments. For each loop structure, a limited range in temporal and spatial domains is identified near the bottom of the loop, where the amplitudes of oscillation are large enough to accurately calculate the phase shift between temperature and density. Within the selected range, the phase shift between the parameters is computed using a cross-correlation method. The obtained values are substantially larger than that expected from the classical Spitzer thermal conductivity. Although this might mean that the thermal conduction is higher than the classical values, one must note that the observed values are even larger than that could be reconciled with the wave theory, which therefore suggests that the apparent phase shifts are not merely due to thermal conduction.

It is also worth noting that most of the observed phase shift values are clustered around $180^{\circ}$ (see Table 1). Jess et al. (2012) studied a sunspot fan-loop structure using an independent DEM method and found that the obtained peak temperature and emission measure are $180^{\circ}$ out of phase. The authors explained this behavior as being due to the anticorrelated changes in emission volume along the line of sight. Although, from our data, we do not find a definitive dependence of phase shifts on the location of the loop structure (perhaps because of the nonuniform distribution of our data with more samples toward the solar disk center), it is possible that a similar effect is responsible for the large phase shifts observed here. For instance, the loop structure that is furthest from the disk center (i.e., from AR11374) in our sample shows the smallest phase shifts $\left(\approx 52^{\circ}\right)$. Besides, the temperature and density values obtained from our DEM analysis are representative of mean values over the cross-section of a loop, which implies the multithermal nature of the active region loops, as evidenced from the differential propagation of slow waves when observed in multiple temperature channels (Kiddie et al. 2012; Uritsky et al. 2013; Krishna Prasad et al. 2017), is not considered. This approximation may also influence the observed phase shifts. It may also be worthwhile exploring whether the misbalance in the local thermal equilibrium caused by the slow waves (Nakariakov et al. 2017) can, in turn, affect the observed phase shifts. Additional effects, such as nonlinearity (Nakariakov et al. 2000;
Ofman \& Wang 2002) and partial wave reflection, can affect the phase shifts but do not seem to be applicable to our data. In any case, even in the absence of the aforementioned effects, the exact phase shift depends on several factors (see Equation (4)) and, hence, should not be assumed to correlate precisely with thermal conduction.

By manually shifting the temperature time series to remove the existing phase shift, we compare the oscillation amplitudes in temperature against that in density and compute the polytropic index. These values occur in the range between $1.04 \pm 0.01$ and $1.58 \pm 0.12$. It also appears that there is a temperature dependence, with hotter loops having a higher polytropic index. Qualitatively, this behavior brings the contrasting findings of Van Doorsselaere et al. (2011) and Wang et al. (2015) to a good agreement. One may note, however, that the observed dependence of polytropic index on temperature (see Figure 5) is steeper than what would be required to have a better match with the previous results. This warrants the requirement of additional examples distributed across a wider temperature range to find the exact dependence. Moreover, the phase shift between the temperature and density perturbations observed in the earlier studies is relatively small $\left(<90^{\circ}\right)$, unlike that in our case. Van Doorsselaere et al. (2011) obtained the temperature and density information directly from the spectroscopic line ratios, so their results are less prone to the line-of-sight effect, which we believe is the cause for the large phase shifts in our data. However, it is intriguing why Wang et al. (2015) do not see any such effect. It is possible that the extremely hot plasma in their loop somehow helps in mitigating the line-of-sight changes, but this requires further studies to confirm and improve our understanding of this effect.

Finally, although it is possible that the increase in polytropic index with temperature might imply a gradual suppression of thermal conduction in agreement with the inferences from previous studies, it is much harder to explain. Wang et al. (2015) offered some explanations based on nonlocal conduction, plasma waves, and turbulence that are more applicable to hot flare loops, but because this behavior appears to be more prevalent even in the warm loops, one needs to find a general theory. Besides, the influence of other thermodynamic processes (e.g., heating and radiative losses), in addition to the observational effects (e.g., multithermal structure of loops) on the polytropic index, should be investigated. We believe that future studies, ideally a combination of observations, numerical simulations, and forward modeling, might reveal important information to address this problem.

The authors thank the anonymous referee for useful comments. S.K.P. is grateful to the UK Science and Technology Facilities Council (STFC) for funding support that allowed this project to be undertaken. T.V.D. was supported by grant No. GOA-2015-014 (KU Leuven) and the European Research Council (ERC) under the European Union's Horizon 2020 Research and Innovation Programme (grant agreement No. 724326). D.B.J. would like to thank STFC for an Ernest Rutherford Fellowship, in addition to Invest NI and Randox Laboratories Ltd. for the award of a Research \& Development Grant (059RDEN-1). AIA data used here are courtesy of NASA/SDO and the AIA science team. We acknowledge the use of pipeline developed by Rob Rutten to extract, process, and coalign AIA cutout data. 


\section{ORCID iDs}

S. Krishna Prasad (i) https://orcid.org/0000-0002-0735-4501

T. Van Doorsselaere (1) https://orcid.org/0000-0001-9628-4113

D. B. Jess $\odot$ https://orcid.org/0000-0002-9155-8039

\section{References}

Berghmans, D., \& Clette, F. 1999, SoPh, 186, 207

Culhane, J. L., Harra, L. K., James, A. M., et al. 2007, SoPh, 243, 19

De Moortel, I. 2009, SSRv, 149, 65

De Moortel, I., \& Hood, A. W. 2003, A\&A, 408, 755

De Moortel, I., Ireland, J., \& Walsh, R. W. 2000, A\&A, 355, L23

De Moortel, I., Ireland, J., Walsh, R. W., \& Hood, A. W. 2002, SoPh, 209, 61

Deforest, C. E., \& Gurman, J. B. 1998, ApJL, 501, L217

Ghosh, A., Tripathi, D., Gupta, G. R., et al. 2017, ApJ, 835, 244

Goossens, M. 2003, An Introduction to Plasma Astrophysics and Magnetohydrodynamics, Vol. 294 (Dordrecht: Kluwer)

Hannah, I. G., \& Kontar, E. P. 2012, A\&A, 539, A146

Houston, S. J., Jess, D. B., Asensio Ramos, A., et al. 2018, ApJ, 860, 28

Jess, D. B., De Moortel, I., Mathioudakis, M., et al. 2012, ApJ, 757, 160

Jess, D. B., Reznikova, V. E., Ryans, R. S. I., et al. 2016, NatPh, 12, 179

Jess, D. B., Van Doorsselaere, T., Verth, G., et al. 2017, ApJ, 842, 59

Kartalev, M., Dryer, M., Grigorov, K., \& Stoimenova, E. 2006, JGRA, 111 A10107

Kiddie, G., De Moortel, I., Del Zanna, G., McIntosh, S. W., \& Whittaker, I. 2012, SoPh, 279, 427

King, D. B., Nakariakov, V. M., Deluca, E. E., Golub, L., \& McClements, K. G. 2003, A\&A, 404, L1

Krishna Prasad, S., Banerjee, D., \& Singh, J. 2012a, SoPh, 281, 67

Krishna Prasad, S., Banerjee, D., \& Van Doorsselaere, T. 2014, ApJ, 789, 118

Krishna Prasad, S., Banerjee, D., Van Doorsselaere, T., \& Singh, J. 2012b, A\&A, 546, A50

Krishna Prasad, S., Jess, D. B., \& Khomenko, E. 2015, ApJL, 812, L15

Krishna Prasad, S., Jess, D. B., Klimchuk, J. A., \& Banerjee, D. 2017, ApJ, 834,103
Kumar, P., Nakariakov, V. M., \& Cho, K.-S. 2015, ApJ, 804, 4

Lemen, J. R., Title, A. M., Akin, D. J., et al. 2012, SoPh, 275, 17

Mandal, S., Yuan, D., Fang, X., et al. 2016, ApJ, 828, 72

Marsh, M. S., \& Walsh, R. W. 2006, ApJ, 643, 540

Marsh, M. S., Walsh, R. W., \& Plunkett, S. 2009, ApJ, 697, 1674

McEwan, M. P., \& de Moortel, I. 2006, A\&A, 448, 763

Nakariakov, V. M., Afanasyev, A. N., Kumar, S., \& Moon, Y.-J. 2017, ApJ, 849,62

Nakariakov, V. M., Verwichte, E., Berghmans, D., \& Robbrecht, E. 2000, A\&A, 362, 1151

Nisticò, G., Polito, V., Nakariakov, V. M., \& Del Zanna, G. 2017, A\&A, 600, A37

Ofman, L., Nakariakov, V. M., \& Deforest, C. E. 1999, ApJ, 514, 441

Ofman, L., Romoli, M., Poletto, G., Noci, G., \& Kohl, J. L. 1997, ApJL, 491, L111

Ofman, L., \& Wang, T. 2002, ApJL, 580, L85

Owen, N. R., De Moortel, I., \& Hood, A. W. 2009, A\&A, 494, 339

Pant, V., Tiwari, A., Yuan, D., \& Banerjee, D. 2017, ApJL, 847, L5

Parker, E. N. 1963, Interplanetary Dynamical Processes (New York: Interscience)

Pesnell, W. D., Thompson, B. J., \& Chamberlin, P. C. 2012, SoPh, 275, 3

Roosen, J. 1969, SoPh, 7, 448

Spitzer, L. 1962, Physics of Fully Ionized Gases (New York: Interscience)

Sun, X., Hoeksema, J. T., Liu, Y., et al. 2013, ApJ, 778, 139

Uritsky, V. M., Davila, J. M., Viall, N. M., \& Ofman, L. 2013, ApJ, 778, 26

Van Doorsselaere, T., Wardle, N., Del Zanna, G., et al. 2011, ApJL, 727, L32

Wang, T. 2011, SSRv, 158, 397

Wang, T., Innes, D. E., \& Qiu, J. 2007, ApJ, 656, 598

Wang, T., Ofman, L., Sun, X., Provornikova, E., \& Davila, J. M. 2015, ApJL, 811, L13

Wang, T., Ofman, L., Sun, X., Solanki, S. K., \& Davila, J. M. 2018, ApJ, 860,107

Wang, T., Solanki, S. K., Curdt, W., Innes, D. E., \& Dammasch, I. E. 2002, ApJL, 574, L101

Wang, T. J., Ofman, L., Davila, J. M., \& Mariska, J. T. 2009, A\&A, 503, L25

Wang, T. J., Solanki, S. K., Innes, D. E., \& Curdt, W. 2005, A\&A, 435, 753 